\title{
Guest Editorial: Real-Time and Embedded Systems
}

\author{
Kaijie $\mathrm{Wu}^{1}$ - Zili Shao ${ }^{2}$
}

Published online: 6 April 2016

(C) Springer Science+Business Media New York 2016

"Real-time and embedded systems" is a fundamental and important research area. While it has a long history, it faces different challenges at different time. This special issue is organized to investigate advances on system design practice and emerging applications, to promote interaction among the areas of embedded and real-time computing, system design practice and emerging applications, to evaluate the maturity and directions of embedded and real-time system, system design practice and emerging applications technology.

Schedulibility is always the first-priority constraint in designing and analyzing real-time systems. In this special issue, we have several papers on this sub topic. They are "Optimal Semi-Partitioned Scheduling in Soft Real-Time Systems" (10.1007/s11265-015-0983-7); "Worst-Case Communication Delay Analysis for NoC-Based ManyCores using a Limited Migrative Model" (10.1007/ s11265-015-0992-6); and "Improved Message Forwarding for Multi-Hop HaRTES Real-Time Ethernet Networks" (10.1007/s11265-015-1010-8).

Power and energy efficiency of embedded systems are gaining more and more attention recently as more and more embedded systems are powered by battery and have limited

Kaijie Wu

kaijie@gmail.com

Zili Shao

cszlshao@comp.polyu.edu.hk

1 Chongqing University, Chongqing, China

2 Hong Kong Polytechnic University, Hung Hom, Hong Kong space for heat dissipation. The following papers are on this sub topic: "Energy Efficient Real-Time Task Scheduling for Embedded Systems with Hybrid Main Memory" (10.1007/ s11265-015-0995-3); "Energy-Efficient Allocation of RealTime Applications onto Single-ISA Heterogeneous MultiCore Processors" (10.1007/s11265-015-0987-3); and "Peak Temperature Minimization via Task Allocation and Splitting for Heterogeneous MPSoC Real-Time Systems" (10.1007/ s11265-015-0994-4).

With the steady progress on the IC design and manufacturing techniques, more and more embedded systems are powered by multi-core CPUs. Exploring various kinds of multi-core architectures and new types of memory has received lots of attention in the past decade. In this special issue we have include the following papers on this sub topic: "Properties of Self-Timed Ring Architectures for DeadlockFree and Consistent Configuration Reaching Maximum Throughput" (10.1007/s11265-015-0984-6), "PUMA: From Simultaneous to Parallel for Shared Memory System in Multi-core" (10.1007/s11265-015-1015-3); and "Data Allocation with Minimum Cost under Guaranteed Probability for Multiple Types of Memories" (10.1007/ s11265-015-0985-5).

Finally, we have a paper that introduces exact and nonexact real-time waits in the reactive Globally Asynchronous Locally Synchronous programming language. The language constructs allow system designers to explicitly use both logical time and real time to control program execution at the specification level. Please find the details in "Times square marriage of real-time and logical-time in GALS and synchronous languages" (10.1007/s11265-015-0991-7).

Finally, we strongly believe that the researchers and engineers who work in this field will find this special issue helpful, and sincerely hope it may stimulate further research. 


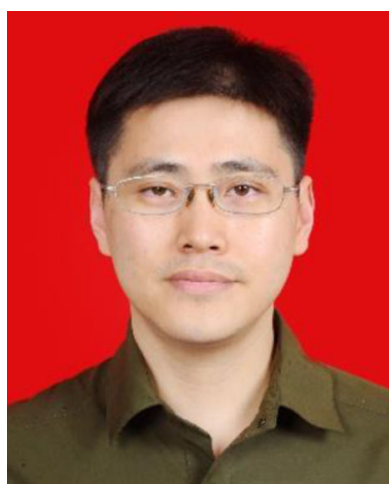

Kaijie Wu received the BE degree from Xidian University, Xi'an, China, in 1996, the MS degree fromthe University of Science and Technology of China, Hefei, China, in 1999, and the Ph.D. degree in electricalengineering from Polytechnic University (Now Polytechnic Institute of New York University), Brooklyn, NewYork, in 2004. He then joined University of Illinois, Chicago, USA as an Assistant Professor. Since 2013 , hebecomes a professor at the College of Computer Science, Chongqing University, China. His research is onthe big area of trustworthy computing with special interest on dependable computing and hardware security.He is the recipient of the 2004 EDAA Outstanding Dissertation Award for "new directions in circuit andsystem test", and the "Most Significant Paper" award from the International Test Conference, 2014. He is amember of the IEEE.

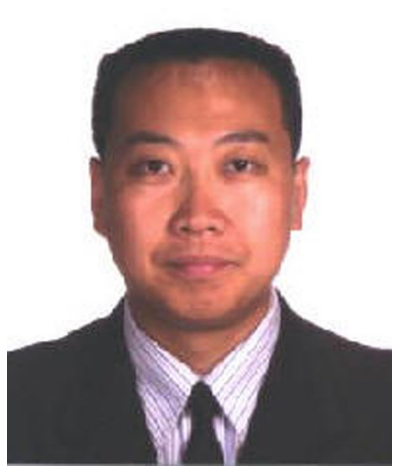

Zili Shao received the BE degree in electronic mechanics from the University of Electronic Science and Technology of China, Sichuan, China, in 1995, and the MS and $\mathrm{PhD}$ degrees from the Department of Computer Science, University of Texas at Dallas, in 2003 and 2005, respectively. He has been an associate professor with the Department of Computing, Hong Kong Polytechnic University, since 2010. His research interests include embedded software and systems, real-time systems, and related industrial applications. 